\title{
Arbitrary Amplitude Ion Acoustic Wave in a Pair Ions and Electron Plasma with Trapped Electrons
}

\author{
Maliheh Sadat Arabjafari, Hossein Zahed", and Elham Emadi \\ Department of Physics, Sahand University of Technology, Tabriz, Iran \\ *Corresponding Author's Email: zahed@sut.ac.ir
}

Regular paper: Received: Sep. 1, 2019, Revised: Apr. 17, 2020, Accepted: May. 10, 2020, Available Online: May. 12, 2020, DOI: 10.52547/ijop.15.1.11

\begin{abstract}
Propagation of ion acoustic solitary waves (IASWs) in electronegative plasma containing positive and negative ions, trapped and non-thermal electrons are investigated. Using the Sagdeev pseudopotential method and investigation of the energy integral, the existence of propagation regions for these waves is analyzed. It is shown that the Mach number, positive and negative ions densities ratio and the trapping parameter can lead to change the pseudopotential amplitude and also it is shown that the lower limit of the Mach number increases with the density and mass ratios of positive and negative ions, but the upper limit of the Mach number does not depend on the densities ratio. The results show that for this kind plasma, there is only compressive IASW. This research will be helpful in understanding of physical phenomena concerned in plasmas in which the effects of trapped electrons control the dynamics of wave.
\end{abstract}

KEYWORDS: Arbitrary amplitude ion acoustic solitary wave, Sagdeev pseudopotential, trapped electron.

\section{INTRODUCTION}

Plasma is an inseparable part of stellar and galactic structure. Using the plasma dynamic equations, the behavior of magnetosphere of the earth and other planets can be described. It forms the research frontier in such areas as fusion, advanced accelerators, and short pulse lasers; and its applications to various industrial processes (such as computer chip manufacture) are rapidly increasing. It is a subject with a large and growing list of scientific and technological applications. The usefulness of plasma physics has, of course, enriched the subject, supporting plasma research programs and inspiring many advances in understanding the plasma state [1].

Most of the researches are interested in un magnetized electron-ion plasmas because enormously it is believed that electron-ion on plasmas may exist in physical space as well as laboratory experiments. Sagdeev first studied the fully nonlinear ion-acoustic waves (IAW) in an unmagnetized plasma consists of cold ions and hot electrons. Recently, the properties of nonlinear IAWs in a two-temperature nonextensive electron plasma have been studied theoretically [2]. So the nature of these nonlinear IAWs has been investigated extensively both experimentally and theoretically [3], [4].

It has been observed that only compressive solitary IAWs involving density hump exist in two-component plasmas. Most of the abovementioned studies discussed up to now are based on the present of Maxwell-Boltzmann distributed components, while numerous observations indicate the deviation of particle distribution functions from the Maxwellian distribution. In this study, we consider arbitrary amplitude IA solitary waves (IASWs) in plasmas with trapped electrons and non-thermal distributions instead of Boltzmann distribution in the phase space. Often, hot electrons are generated [5]-[7] in the astrophysical and cosmic plasmas, but the minority heavy ions and cold electrons are probably present, as observed in the outflows of electrons plasmas from the pulsars entering into cold and low- 
density electron-ion interstellar plasma, the phenomena form two temperature e-i plasmas. In such plasmas, electrons that have distinct temperature and high density have a great chance to be in distinction with IAWs and be trapped. The electron trapping has been observed commonly in both space and laboratory plasma [8]-[10].

Theory of electron trapping by an IASW was proposed by H. Schamel [5], [11], [12]. Several authors experimentally [9], [13]-[16] have studied the effect of trapped electrons on plasmas. Schamel et al. [10] showed that electrons with lower kinetic energy level are trapped in the nonlinear wave potential and the electrons confined by the wave potential, and so they oscillate in a region of the phase space. Alinejad et al. [17] have studied largeamplitude IASW in a plasma with cold ions, hot positrons, and electrons that have trapped particles. They showed that the positron density can change the maximum possible value of the amplitude and Mach number for the existence of solitary waves [17]. Nakamura et al. [12] studied experimentally IASWs in a threecomponent plasma consisting of positive and negative ions and electrons. Recently, Siham Ghebache et al. discussed the properties of IAWs in one dimensional electronegative plasma, consisting of positive and negative ions and nonextensive electrons. This study has shown that in such a plasma model, smooth rarefactive and spiky compressive IASWs can exist. To the best of our knowledge, no one investigated the nonlinear propagation of IAW in an unmagnetized one-dimensional plasma system consisting of positive and negative ions and trapped electrons. Hence, in this article, we study the properties of nonlinear IAWs by using the arbitrary amplitude method and the pseudopotential approach.

The article is organized as follows: in section II, basic equations are given and numerical results and discussion are presented in section III. And finally, section IV is kept for conclusions.

\section{BASIC EQUATIONS}

We investigate arbitrary amplitude IA solitons supported in a one-dimensional, collisionless and unmagnetized plasma system consisting of trapped and non-thermal electrons, and cold positive ions (negative ions) which is shown with subscript $i(n)$. The unperturbed densities of the electron $\left(n_{e}\right)$, cold positive ion $\left(n_{i 0}\right)$, and cold negative ions $\left(n_{n 0}\right)$ are considered to maintain the charge neutrality equilibrium condition $n_{e 0}+n_{n 0}=n_{i 0}$. The dynamics of IA oscillations is governed by the following system of equations:

$\frac{\partial n_{i}}{\partial t}+\frac{\partial\left(n_{i} u_{i}\right)}{\partial x}=0$

$\frac{\partial u_{i}}{\partial t}+u_{i} \frac{\partial u_{i}}{\partial x}=-\frac{\partial \Phi}{\partial x}$

$\frac{\partial n_{n}}{\partial t}+\frac{\partial\left(n_{n} u_{n}\right)}{\partial x}=0$

$\frac{\partial u_{n}}{\partial t}+u_{n} \frac{\partial u_{n}}{\partial x}=G \frac{\partial \Phi}{\partial x}$

where $G=m_{i} / m_{n}$ and $m_{i}\left(m_{n}\right)$ is the positive (negative) ion mass. The expression for the trapped electrons density is given by [1]-[4]:

$n_{e}=\exp \Phi \operatorname{erfc}(\sqrt{\Phi})+\frac{\exp \beta \Phi}{\sqrt{\pi|\beta|}}$,

$\operatorname{erf}(\sqrt{\beta \Phi}), \beta \geq 0$

where $\operatorname{erfc}(\Phi)=1-\operatorname{erf}(\Phi)$ and $\beta=T_{e} / T_{e t}$ (ratio of the free electron temperature to trapped electron temperature). The role of $\beta$ is to change the depth of the distribution function hole.

By keeping the terms up to $\Phi^{2}$ and expanding $n_{e}$ for the small-amplitude limit, i.e., $\Phi \leq 1$, we obtain [1], [5]:

$$
n_{e}=1+\Phi-\frac{4}{3} a \Phi^{3 / 2}+\frac{1}{2} \Phi^{2}
$$

where $a=\pi^{-1 / 2}(1-\beta)$ measures the deviation from isothermality condition. The term $a>0$ is the contribution share of the resonant electrons to the electron density. 
To make this system self-consistent, Poisson's equation will be applied in the following form:

$\nabla^{2} \Phi=n_{n}-n_{i}+n_{e}$

Charge neutrality condition implies that $n_{n 0} / n_{i 0}+n_{e 0} / n_{i 0}=1$, where $\delta=n_{n 0} / n_{i 0}$. The positive (negative) ion density $n_{i}\left(n_{n}\right)$ is normalized by using $n_{i 0}$, the positive (negative) ion fluid velocity $u_{i}\left(u_{n}\right)$ is normalized by using IA speed $C_{s}=\left(T_{e} / m_{i}\right)^{1 / 2} \quad\left(T_{e}\right)$ is the free electron temperature). And also, the electrostatic wave potential $(\Phi)$, time variable $(t)$ and space variable $(x)$ are normalized by, $T_{e} / e$ ( $e$ is the electron base charge), the period of ion plasma $\omega_{i}^{-1}=\left(4 \pi n_{e 0} e^{2} / m_{i}\right)^{1 / 2}$ and ion Debye radius, $\quad \lambda_{D i}=\left(\mathrm{T}_{e} / 4 \pi n_{i 0} e^{2}\right)^{1 / 2}$, respectively.

To study the characteristics of the solitary wave solutions, we use the transformation $\xi=x-M t$, where $M$ is the Mach number (the velocity of the solitary wave), and $\xi$ is normalized by $\lambda_{D i}$. Applying this transformation under the appropriate boundary conditions, namely, $\Phi \rightarrow 0, u_{i, n} \rightarrow 0, n_{i} \rightarrow 1$, and $n_{n} \rightarrow \delta$ at $\xi \rightarrow \pm \infty$, on Eqs. (1)-(6) gives:

$-M \frac{\partial n_{i}}{\partial \xi}+\frac{\partial\left(n_{i} u_{i}\right)}{\partial \xi}=0$

$-M \frac{\partial u_{i}}{\partial \xi}+u_{i} \frac{\partial u_{i}}{\partial \xi}+\frac{\partial \Phi}{\partial \xi}=0$,

$-M \frac{\partial n_{n}}{\partial \xi}+\frac{\partial\left(n_{n} u_{n}\right)}{\partial \xi}=0$,

$-M \frac{\partial u_{n}}{\partial \xi}+u_{n} \frac{\partial u_{n}}{\partial \xi}-G \frac{\partial \Phi}{\partial \xi}=0$,

$\frac{\partial^{2} \Phi}{\partial^{2} \xi}=n_{n}-n_{i}+n_{e}$

After integrating Eqs. (11) and (12) and applying appropriate Dirichlet boundary conditions for localized perturbations, viz.,
$\Phi \rightarrow 0, u_{i, n} \rightarrow 0, n_{i} \rightarrow 1$, and $n_{n} \rightarrow \delta$ at $\xi \rightarrow \pm \infty$, we write:

$-M n_{i}+n_{i} u_{i}=M$,

$u_{i}=M\left(1-\frac{1}{n_{i}}\right)$

and

$-M u_{i}+\frac{u_{i}^{2}}{2}=-\Phi$,

Substituting Eq. 13 into Eq. 14, we have:

$n_{i}=\frac{1}{\sqrt{1-\frac{2 \Phi}{M^{2}}}}$,

Similarly, using Eqs. (9) and (10), we obtain for negative ion number density $n_{n}$ as:

$$
n_{n}=\frac{\delta}{\sqrt{1+\frac{2 G \Phi}{M^{2}}}},
$$

Substituting the value of $n_{i}, n_{n}$ and $n_{e}$ into Poisson's equation, the energy equation for quasi-particle is determined as follows:

$$
\begin{aligned}
& \frac{d^{2} \Phi}{d \xi^{2}}=\exp \Phi \operatorname{erfc}(\sqrt{\Phi})+ \\
& \frac{\exp \beta \Phi}{\sqrt{\pi|\beta|}} \operatorname{erf}(\sqrt{\beta \Phi})^{2}- \\
& \frac{1}{\sqrt{1-\frac{2 \Phi}{M^{2}}}}+\frac{\delta}{\sqrt{1+\frac{2 G \Phi}{M^{2}}}}
\end{aligned}
$$

We derive the following equation known as energy integral with $V(\Phi)$ as Pseudopotential (or Sagdeev potential) [6]:

$\frac{1}{2}\left(\frac{d \Phi}{d \xi}\right)^{2}+V(\Phi)=0$,

where: 


$$
\begin{aligned}
& V(\Phi)=1-\exp \Phi \cdot \operatorname{erfc}(\sqrt{\Phi}) \\
& -\frac{\exp \beta \Phi \cdot \operatorname{erf}(\sqrt{\beta \Phi})}{\sqrt{\beta^{3}}}+ \\
& \frac{2 \sqrt{\Phi}(1-\beta)}{\beta \sqrt{\pi}}+M^{2}\left[\left(1-\frac{2 \Phi}{M^{2}}\right)^{1 / 2},\right. \\
& \left.-1-\frac{\delta}{G}+\frac{\delta}{G}\left(1+\frac{2 G \Phi}{M^{2}}\right)^{1 / 2}\right]
\end{aligned}
$$

It is revealed from Eq. 19 that $\left.V^{\prime}(\Phi)\right|_{\Phi=0}=0$. Therefore, the solitary wave solution of Eq. (18) exists if:

(i) $\left.V^{\prime \prime}(\Phi)\right|_{\Phi=0}<0$, so that the fixed point at the origin has unstable condition.

(ii) there exists a nonzero $\Phi_{m}$, the maximum (or minimum) value of $\Phi$, for which $V\left(\Phi_{m}\right)=0$.

(iii) $V(\Phi)<0$, when $\Phi$ lies in the range $0<\Phi<\Phi_{m}$.

The critical Mach number, at which the second derivative changes its sign, can be found from the first condition as:

$$
M>M_{\min }=\sqrt{1+G \delta},
$$

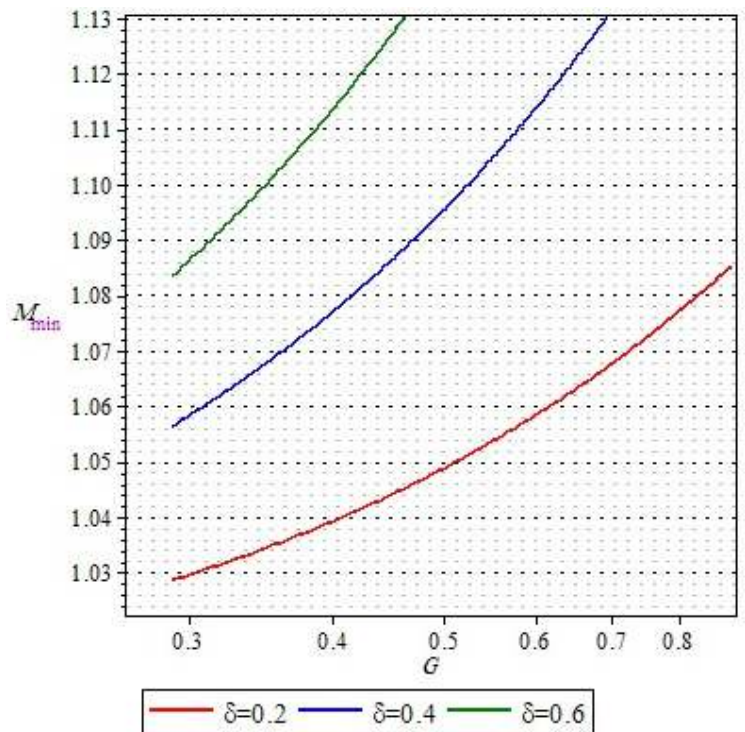

Fig. 1. The behavior of the Mach number $M$ on the ion mass ratio $G$ for different value of $\delta$.
This clearly indicates that increasing $G$, the positive to negative ion mass ratio, leads to increasing critical Mach number $M_{\min }$. It is also indicated that the lower limit of the Mach number $M_{\min }$ strongly depends on the density ratio, $\delta=n_{n 0} / n_{i 0}$. (See Fig. 1)

On the other hand, to find the upper limit of the Mach number, $M_{\max }$, we follow the model of $S$. Sultana et al. [7] and Siham Ghebache et al. [8] who have used the condition $V\left(\Phi_{c}\right) \geq 0$ (where $\Phi_{c}=M_{2} / 2$ is the maximum value of $\Phi$ for which the cold positive ion density $n_{i}$ can be real), to investigate the existence of $M_{\max }$. Thus, we obtain:

$$
\begin{aligned}
& \mathrm{V}\left(\Phi_{c}\right)=1-\exp \left(M^{2} / 2\right) \\
& \operatorname{erfc}\left(\sqrt{M^{2} / 2}\right)-\frac{1}{\sqrt{\beta^{3}}} \exp \left(\beta M^{2} / 2\right) \\
& \operatorname{erf}\left(\sqrt{\beta M^{2} / 2}\right)+\frac{\sqrt{2 M^{2}}}{\beta \sqrt{\pi}}(1-\beta), \\
& +M^{2}\left[1+\frac{\delta}{G}(1-\sqrt{1+G})\right] \geq 0 .
\end{aligned}
$$

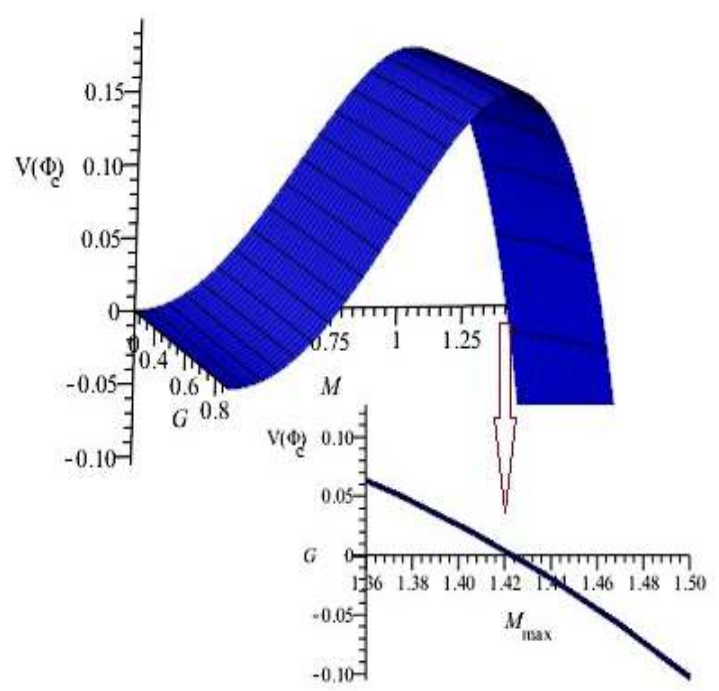

Fig. 2. Behavior of $V\left(\Phi_{c}\right)$ against the Mach number, $M$, and the ion mass ratio, $\mathrm{G}$, for different value of $\delta$ $(\delta=0.2, \delta=0.4, \delta=0.6)$ and $\beta=0.8$.

In Fig. 2. $V\left(\Phi_{c}\right)$ is plotted against $M$ and $G$ for different values of $\delta$. This shows how the upper limit of the Mach number, $M_{\max }$, changes in accordance with ion mass ratio when other 
physical parameters are held constant. For given different values of the density ratio, behavior of upper limit of the Mach number is similar to the previous one, hence the upper limit of the Mach number is independent of density ratio, $\delta$.

\section{III.NUMERICAL RESULTS AND DISCUSSION}

The Sagdeev pseudopotential $V(\Phi)$ plays a significant role in the formation of solitary waves, hence it is essential to study dynamics of solitons as a function of plasma parameters such as $M, \delta, G$ and $\beta$. Fig. 3 shows the behavior of arbitrary IASWs in terms of different values of Mach number $M$. It is observed that increasing the Mach number leads to an increase in the pseudopotential amplitude and also its width. We find that compressive solitons are created, but rare active solitons tend not to exist. The efficacy of the ion mass ratio, $G$, on the Sagdeev pseudopotential is shown in Fig. 4. It is demonstrated that increasing $G$ can lead to a decrease in the pseudopotential amplitude but the width of pseudopotential remains stable. It is concluded that only compressive solitons can be created. The behavior of $\beta$ on the pseudopotential $V(\Phi)$ plotted in Fig. 5 for $M=1.1, G=0.27$, and $\delta=0.4$, which depicts that when the $\beta$ increases, a potential well curve on the positive $\Phi$ axis is created that can be lead to the creation of compressive solitary waves.

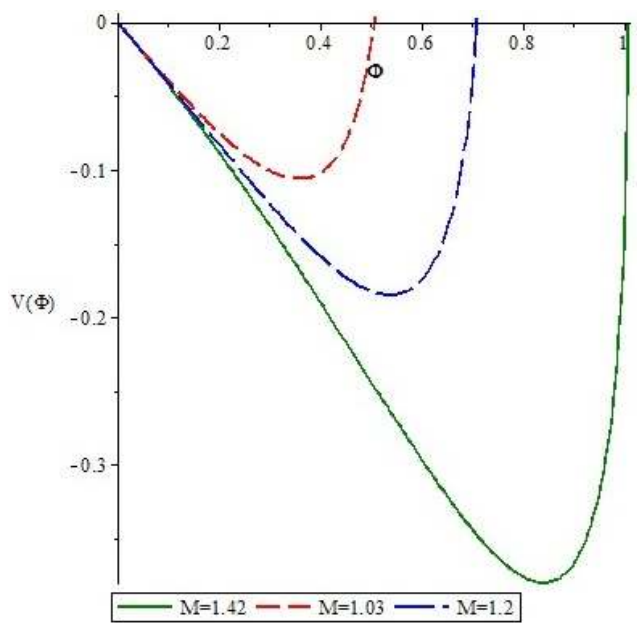

Fig. 3. The effect of $M$ on the Sagdeev pseudopotential curve for fixed values: $\delta=0.4, \beta=0.8$ and $G=0.27$

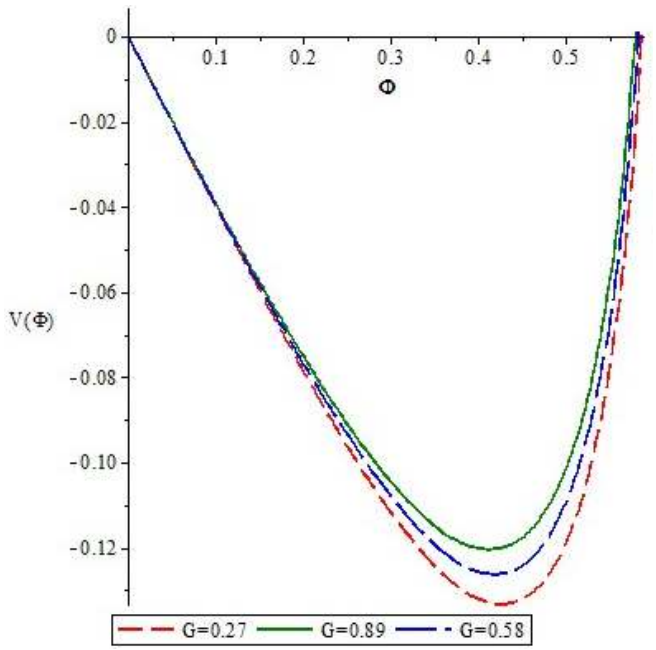

Fig. 4. The behavior of $G$ on the Sagdeev pseudopotential curve for fixed values: $M=1.1$, $\beta=0.8$ and $\delta=0.4$.

Figure 6 displays the plot of $V(\Phi)$ against $\Phi$ for different values of the density ratio $\delta$. A small increase in $\delta$ results in the existence of wells in the Sagdeev pseudopotential for positive potential values $(\Phi>0)$, signifying the existence of spatially localized (soliton-like) compressive IASWs. Fig. 7 displays the behavior of $V(\Phi)$ against $\Phi$ and $G$ for different values of the Mach number $M$.

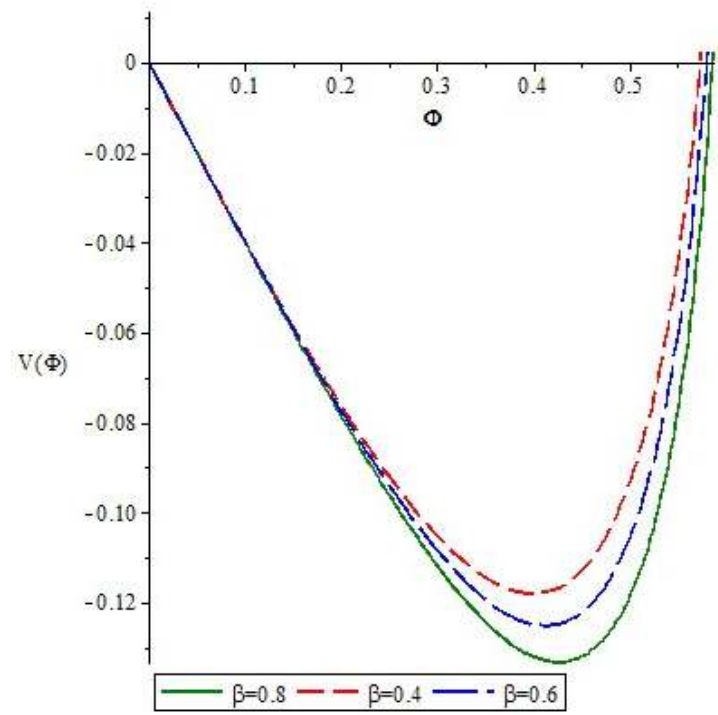

Fig. 5. The behavior of Sagdeev pseudopotential curve as a function of $\beta$ for fixed values $M=1.1$, $\mathrm{G}=0.27$ and $\delta=0.4$

It shows that increasing $M$ leads to an increase in the amplitude and width of the pseudopotential. It is clearly observed from Fig. 
6 that even a small increase in $\delta$ can be lead to enhance the pseudopotential amplitude.

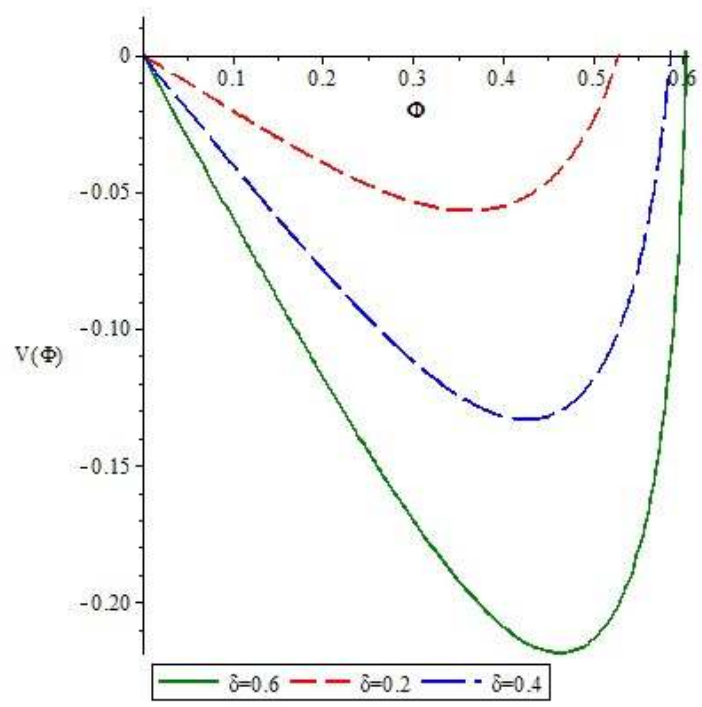

Fig. 6. The behavior of $\delta$ on the Sagdeev pseudopotential curve for fixed values: $M=1.1$, $\beta=0.8$ and $G=0.27$

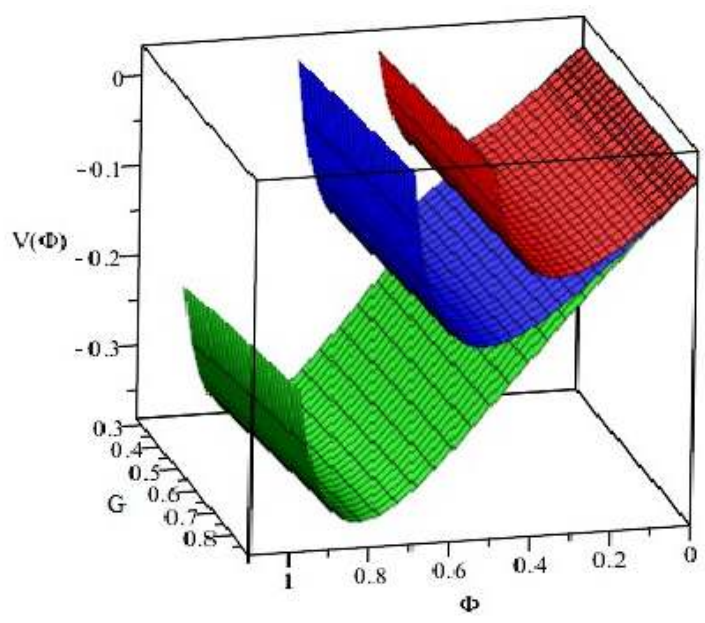

Fig. 7. The behavior of $\Phi$ and $G$ on the Sagdeev pseudopotential curve for fixed values: $M=1.03$ (Red), $M=1$ (blue), $M=1.42$ (green), $\beta=0.8, \delta=0.4$.

\section{IV.CONCLUSION}

Using the Sagdeev pseudopotential, the arbitrary amplitude IA solitons were investigated in the presence of trapped electrons.

It was found that increasing the Mach number, $M$, density ratio, $\delta$, and trapping parameter, $\beta$, increased the pseudopotential amplitude. However, increasing the ion mass ratio, $G$, decreased the pseudopotential amplitude. It was also observed that increasing the Mach number and the density ratio increased the pseudopotential width. Additionally, it was seen that only positive potentials can exist. Interestingly, it was found that our plasma model supports the existence of compressive ion acoustic solitary waves (IASWs). It was shown that the possible minimum value of Mach number increased as density ratio $\delta$ and $G$ grow, but the possible maximum value of Mach number did not depend on density ratio, $\delta$, but it depends on the ion mass ratio, G. Our present plasma model data is inspired by the experimental studies of Ichiki et al. [9], therefore, the results of this model may be helpful to understanding significant features of this kind of plasma in space and laboratory.

\section{REFERENCES}

[1] H. Alinejad, "Non-linear localized ion-acoustic waves in electron-positron-ion plasmas with trapped and non-thermal electrons," Astrophys. Space Sci. Vol. 325, pp. 209-215, 2010.

[2] H. Schamel, "A modified Korteweg-de Vries equation for ion acoustic wavess due to resonant electrons," Phys. Plasmas, Vol. 9, pp. 377-387, 1973.

[3] H. Schamel, "Analytic BGK modes and their modulational instability," Phys. Plasmas, Vol. 13, pp. 139-145, 1975.

[4] H. Schamel, "Role of trapped particles and waves in plasma solitons-theory and application," Phys. Scr. Vol. 20, pp. 306 (1-12), 1979.

[5] S. Guo, L. Mei, Y.-L. He, H. Guo, and Y. Zhao, "The effect of trapped electrons on the threedimensional ion-acoustic shock wave in magnetized ionic-pair plasma," EPL (Europhys. Lett.), Vol. 114, pp. 25002 (1-6), 2016.

[6] I.B. Bernstein, J.M. Greene, and M.D. Kruskal, "Exact nonlinear plasma oscillations," Phys. Rev. Vol. 108, pp. 546, 1957.

[7] S. Sultana and R. Schlickeiser, "Arbitrary amplitude nucleus-acoustic solitons in multiion quantum plasmas with relativistically degenerate electrons," Phys. Plasmas, Vol. 25, pp. 022110 (1-7), 2018. 
[8] S. Ghebache and M. Tribeche, "Arbitrary amplitude ion-acoustic solitary waves in electronegative plasmas with electrons featuring Tsallis distribution," Phys. A: Stat. Mech. Appl. Vol. 483, pp. 193-200, 2017.

[9] R. Ichiki, M. Shindo, S. Yoshimura, T. Watanabe, and Y. Kawai, "Ion acoustic waves in one-and two-negative ion species plasmas," Phys. Plasmas, Vol. 8, pp. 4275-4283, 2001.
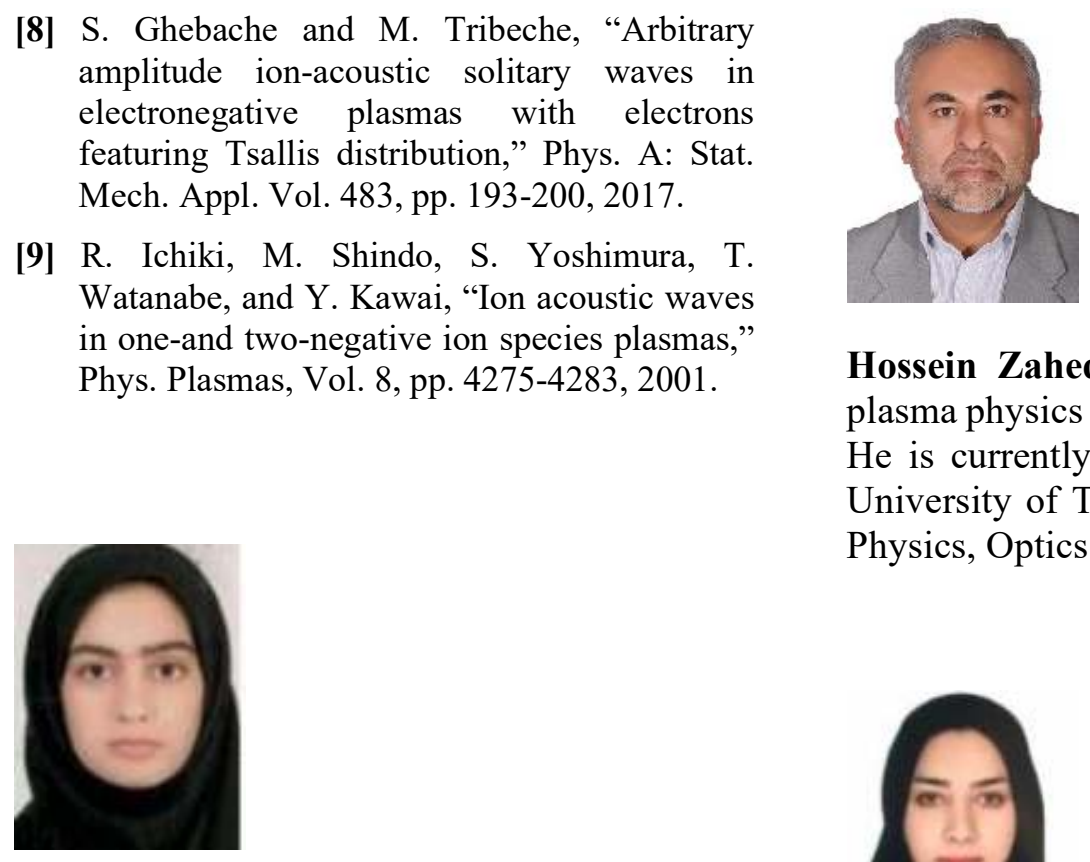

Hossein Zahed received his $\mathrm{PhD}$ degree in plasma physics from Tabriz University in 2006. $\mathrm{He}$ is currently associate professor at Sahand University of Technology, He is a member of Physics, Optics and Photonics Society of Iran.

Maliheh Sadat Arabjafari received her M.Sc. degree in plasma physics from Sahand University of Technology in 2017. She is a member of Physics, Optics and Photonics Society of Iran.

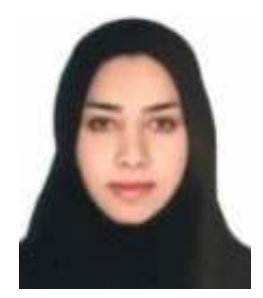

Elham Emadi received her M.Sc. degree in plasma physics from Sahand University of Technology in 2014. She is currently Ph.D. student at Sahand University of Technology. She is a member of Physics, Optics and Photonics society of Iran. 
THIS PAGE IS INTENTIONALLY LEFT BLANK. 